\title{
Flutuação populacional do percevejo-do-grão, Oebalus spp. (Hemiptera: Pentatomidae), em Santa Catarina
}

\author{
Eduardo Rodrigues Hickel' e Douglas George de Oliveira²
}

\begin{abstract}
Resumo - Os percevejos-do-grão são pragas de difícil controle em arroz irrigado. Em Santa Catarina ocorrem Oebalus poecilus (Dallas) e Oebalus ypsilongriseus (DeGeer) (Hemiptera: Pentatomidae). O conhecimento da flutuação populacional dessas espécies permitiria equacionar o controle químico conforme os preceitos do manejo integrado de pragas. Desta forma, objetivou-se monitorar a atividade de voo de Oebalus spp. para conhecer a flutuação populacional e determinar as épocas de maior ocorrência das espécies e morfologias nas lavouras. Armadilhas luminosas foram instaladas em áreas de cultivo de arroz irrigado na Estação Experimental da Epagri em Itajaí, SC e no Centro de Treinamento da Epagri, em Araranguá, SC. A partir de setembro de 2008 a abril de 2020, as armadilhas foram ligadas das 16 às 9 horas, uma vez por semana, exceto de maio a agosto de cada ano, quando permaneceram desligadas. A flutuação populacional do percevejo-do-grão em arroz irrigado caracteriza-se por dois momentos de maior incidência de indivíduos, em novembro/dezembro e em fevereiro, correspondendo aos períodos de ocorrência de percevejos hibernados e dos enxames de verão, respectivamente. A espécie Oebalus poecilus (Dallas) prevalece sobre Oebalus ypsilongriseus (DeGeer) e as populações com morfologias hibernal ou estival ocorrem alternadamente ao longo da safra, sobrepondo-se apenas a partir de meados de fevereiro.
\end{abstract}

Termos para indexação: ecologia; dinâmica populacional; manejo de pragas; Oryza sativa.

\section{Population dynamics of rice stink bugs, Oebalus spp. (Hemiptera: Pentatomidae), in Santa Catarina state, Brazil}

\begin{abstract}
The rice stink bugs are pests of difficult control in irrigated rice. In Santa Catarina, Oebalus poecilus (Dallas) and Oebalus ypsilongriseus (DeGeer) (Hemiptera: Pentatomidae) predominate. The knowledge of the population dynamics of these species, would allow to proper timing the chemical control according to the precepts of integrated pest management. The aim of this study was monitoring the rice stink bugs flight activity to know their population dynamics and the periods of greatest occurrence of species and morphologies in rice fields. Light traps, were set in irrigated rice fields at Epagri Experimental Station, in Itajaí, SC and at Epagri Training Center, in Araranguá, SC. From September 2008 to March 2020 the light traps were turned on, from $16 \mathrm{pm}$ to 9am once a week, except from May to August of each year when they remained turned off. The population dynamics of the rice stink bug in irrigated rice is characterized by two moments of greater bug catches, in November/December and in February, corresponding to the periods of occurrence of hibernates and summer stink bug swarms, respectively. The species Oebalus poecilus (Dallas) prevails over Oebalus ypsilongriseus (DeGeer) and populations with winter or summer morphologies occur alternately throughout the season, only overlapping from mid-February.
\end{abstract}

Index terms: ecology; pest occurrence; integrated pest management; Oryza sativa.

\section{Introdução}

Os percevejos-do-grão são pragas frequentes do arroz irrigado no Brasil. Duas espécies ocorrem nas lavouras, sendo que Oebalus poecilus (Dallas) prevalece sobre Oebalus ypsilongriseus (DeGeer) (FERREIRA et al., 2001; COUTO et al., 2006). Estas duas espécies diferenciam-se pela quantidade de manchas amarelas no dorso e pelo formato do corpo. O. ypsilongriseus tende a ser mais esguio e de coloração geral mais clara, sendo que as manchas amarelas do dorso formam a letra Y (BARRIGOSSI, 2008). Os hábitos e a biologia dessas espécies são similares e, portanto, foram considerados em conjunto nesta pesquisa.

Espécies de Oebalus (Hemiptera: Pentatomidae) causam danos em lavouras de arroz irrigado por todo o continente americano. Oebalus pugnax (F.) é a principal espécie norte-americana, com ampla distribuição nos Estados Unidos e em Cuba (RASHID et al., 2006). Em Cuba, também ocorre com igual severidade Oebalus insularis (Stål) (ARIAS \& GUTIERREZ, 1986). Oebalus grisescens (Sailer) é a espécie difundida pela América Central e Oebalus ornatus (Sailer) ocorre na Colômbia e norte da América do Sul (PANTOJA et al., 2000).

Tanto em $O$. poecilus como em $O$. ypsilongriseus ocorre dimorfismo morfológico em função da época do ano,

Recebido em 26/8/2020. Aceito para publicação em 30/10/2020.

${ }^{1}$ Engenheiro-agrônomo, Dr., Epagri / Estação Experimental de Itajaí, C.P. 277, 88301-970 Itajaí, SC, fone: (47) 3398-6337, e-mail: hickel@epagri.sc.gov.br

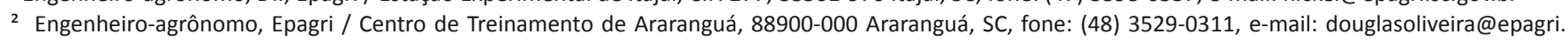
sc.gov.br 
desencadeado pelo fotoperíodo no desenvolvimento ninfal (ALBUQUERQUE, 1993). Isto resulta na existência de adultos estivais: maiores, mais coloridos e com espinhos torácicos salientes; e adultos hibernantes: menores, de coloração dorsal predominante marrom e cujos espinhos são curtos e arredondados ou até ausentes. A diapausa ocorre nos meses de baixa temperatura e de ausência de gramíneas hospedeiras (ALBUQUERQUE, 1993; SANTOS et al., 2006).

O ciclo biológico completa-se em cerca de 45 dias, sendo o período ninfal em torno de 40 dias e 5 dias a incubação dos ovos. As ninfas passam por cinco estágios de desenvolvimento, sendo o primeiro estágio completado em 3 dias e os seguintes em 7, 8, 9 e 13 dias, respectivamente (AMARAL, 1949; FERREIRA et al., 2001). Em laboratório, a longevidade média de adultos acasalados é de 101 dias para os machos e 88 dias para as fêmeas. Entretanto, para os adultos das gerações estivais, a longevidade é menor, em média 35 dias. Fêmeas com intensa atividade de postura vivem em média 14 dias e morrem 8 dias após a última postura (FERREIRA et al., 2001). Estima-se que, durante a permanência do arroz no campo, de duas a três gerações do percevejo se completem. Contudo, como os insetos que chegam ao arroz já são de gerações estivais, acredita-se que outras duas ou três ocorreram anteriormente na vegetação nativa (DOUGLAS, 1939; FERREIRA et al., 2001).

No Sul do Brasil, os registros da flutuação populacional de Oebalus spp. no período de cultivo do arroz são escassos, o que dificulta o desenvolvimento e a adoção de novas estratégias para o manejo da praga, bem como a racionalização da aplicação de inseticidas (HICKEL et al., 2016).

O percevejo-do-grão apresenta fototropismo positivo, o que viabiliza o emprego de armadilhas luminosas para os estudos de flutuação populacional.
Assim, foi objetivo desta pesquisa monitorar a atividade de voo de Oebalus spp. para conhecer a flutuação populacional e determinar as épocas de maior ocorrência das espécies e morfologias nas lavouras.

\section{Material e métodos}

O estudo foi conduzido desde a safra 2008/09, na Estação Experimental da Epagri (EEI), em Itajaí, SC, inicialmente em duas quadras de arroz irrigado, uma de 0,15 ha (quadra $\mathrm{C} 1,26^{\circ} 56^{\prime} 44^{\prime \prime} \mathrm{S}$ e $48^{\circ} 45^{\prime} 42^{\prime \prime}$ ) e outra de 0,24 ha (quadra F8, $26^{\circ} 56^{\prime} 38^{\prime \prime}$ S e $48^{\circ} 45^{\prime} 31^{\prime \prime}$ )). A partir da safra 2015/16, foi incorporada ao estudo outra quadra, de 0,38 ha (quadra $F 1,26^{\circ} 56^{\prime} 44^{\prime \prime} S$ e $\left.48^{\circ} 45^{\prime} 31^{\prime \prime} O\right)$. Estas quadras estão inseridas na área experimental de arroz irrigado de 11,5ha da EEI. Entre as safras 2012/13 e 2015/16, o estudo também foi conduzido na área de arroz irrigado de 25ha do Centro de Treinamento da Epagri em Araranguá, SC, (quadra Cetrar, $28^{\circ} 55^{\prime} 53^{\prime \prime} \mathrm{S}$ e $\left.49^{\circ} 29^{\prime} 52^{\prime \prime} \mathrm{O}\right)$, cerca de $300 \mathrm{~km}$ ao sul de Itajaí.

Em todas as safras e quadras da EEI, o sistema de cultivo adotado foi o prégerminado, conforme preconizado por Eberhardt \& Schiocchet (2015), porém sem a aplicação de inseticidas. No "Cetrar", foi praticado o cultivo orgânico em sistema pré-germinado, conforme preconizado por Noldin et al. (2015). As semeaduras ocorreram sempre no primeiro decêndio de setembro na quadra C1 e no último decêndio de setembro nas quadras F1 e F8. Na quadra Cetrar a semeadura foi sempre no primeiro decêndio de novembro.

Armadilhas luminosas, modelo "Luiz de Queiroz" com luz negra (T8 15W BL $L E)$, foram suspensas em postes de concreto, na altura ${ }^{3}$ de $1,5 \mathrm{~m}$ do solo, sendo duas posicionadas nas quadras $\mathrm{F} 1 \mathrm{e} \mathrm{Ce}-$ trar e uma na F8. Na quadra F1 foi instalada, em tripé metálico, uma armadilha luminosa solar "Sonne", equipada com lâmpada de 3W de LEDs azuis e UVs (ul- travioleta) (KNABBEN et al., 2019). As armadilhas foram posicionadas ao lado da taipa, na metade do maior comprimento da quadra. A armadilha adicional da quadra C1 foi posicionada no canto nordeste. Para limitar a entrada de insetos maiores, uma tela de náilon (10 $\times 10 \mathrm{~mm}$ de malha) foi colocada circundando as aletas das armadilhas.

Anualmente, no período de 30/07 a $27 / 04$, as armadilhas foram ligadas das 16 às 9 horas uma vez por semana, ficando inoperantes na entressafra (maio a agosto). Os insetos atraídos foram aprisionados em sacos plásticos de 20L, fixados no funil coletor da armadilha, de onde posteriormente efetuou-se a triagem e contagem dos percevejos. A partir da safra 2016/17, as capturas de percevejo nas armadilhas da EEI foram discriminadas pela espécie e morfologia corporal, conforme os descritores em Albuquerque (1993) e Barrigossi (2008). Com o registro das contagens foram confeccionados os gráficos de flutuação populacional, bem como estabelecidos os eventuais períodos de maior ocorrência no campo, tanto de espécies como de morfologias corporais. Para o cálculo das médias de capturas de indivíduos, as datas nas diferentes séries temporais foram padronizadas, de acordo com os períodos semanais de cada mês.

\section{Resultados e discussão}

A flutuação populacional do percevejo-do-grão em Santa Catarina é apresentada na Figura 1. Conforme salientam Hickel et al. (2019), há dois períodos de maior ocorrência de indivíduos. O primeiro, entre meados de novembro e dezembro, corresponde à movimentação dos hibernados, ou seja, aqueles adultos que passaram o inverno em diapausa e, nesse período, abandonam os sítios de hibernação (SANTOS et al., 2006). O segundo momento, durante o mês de fevereiro em Itajaí e do final de fevereiro ao início de abril em Araranguá, corresponde ao período dos»

${ }^{3}$ Altura entre o nível do solo e a abertura do funil coletor da armadilha luminosa. 
enxames, quando grandes quantidades de indivíduos se dispersam entre as lavouras (ALBUQUERQUE, 1993; COUTO et al., 2006). Esse segundo momento coincide com boa parte das lavouras no estágio de enchimento de grãos, mais suscetível ao dano pelos percevejos ( $\mathrm{HI}$ CKEL et al., 2016; 2019).

Em Araranguá, a população de percevejos que ocorre a partir de meados de fevereiro é de vinte a cinquenta vezes maior que a registrada em Itajaí. Isto resulta do cultivo orgânico praticado no Cetrar, que, por formar os grãos mais tarde, atrai toda a população de percevejos das lavouras de entorno, já colhidas ou em fase de colheita (HICKEL et al., 2016; 2019).

Couto et al. (2006) reportam flutuação populacional similar para 0 . poecilus e 0 . ypsilongriseus em São Miguel do Araguaia (Goiás), com as maiores populações ocorrendo em novembro e depois entre janeiro e fevereiro. Vivas et al. (2010) também registraram dois picos de ocorrência para adultos de $O$. insularis na Venezuela, sendo o primeiro em novembro, porém o segundo entre maio e junho; mas ambos coincidentes com a fase reprodutiva do arroz. $\mathrm{Na}$
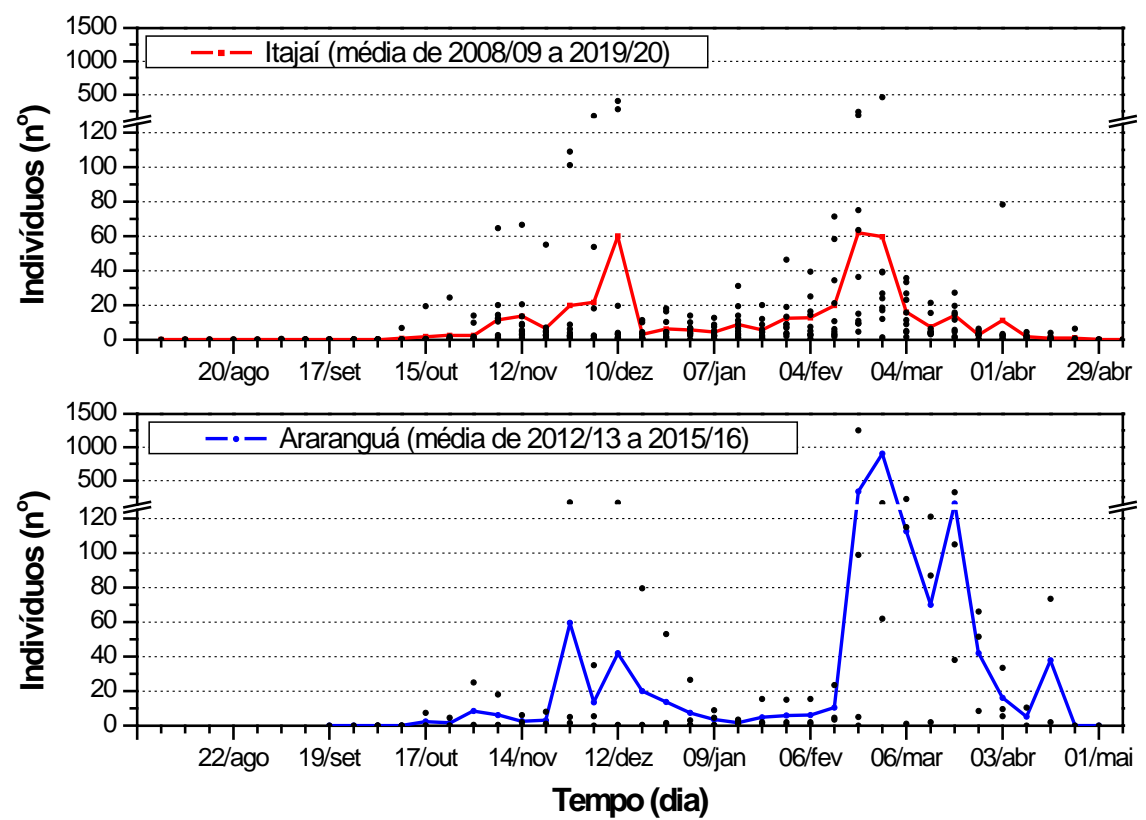

Figura 1. Flutuação populacional média de adultos de Oebalus spp. em Itajaí, SC, safras de 2008/09 a 2019/20 e em Araranguá, SC, safras de 2012/13 a 2015/16

Figure 1. Average adult population dynamics of Oebalus spp. in Itajaí, SC, 2008/09 to 2019/20 seasons and in Araranguá, SC, 2012/13 to 2015/16 seasons rede de varredura, nesse período, não revela a real população de percevejos, pois poucos indivíduos estão pelas áreas de lavoura. Com armadilhas atrativas, a flutuação populacional de O. pugnax tem igualmente dois picos, porém o primeiro ocorre entre junho e julho, em hospedeiros alternativos (durante o ciclo vegetativo do arroz no hemisfério norte); e o segundo entre agosto e setembro, na lavoura de arroz (RASHID et al., 2006).

A habilidade dos percevejos Oebalus formarem enxames só ocorre sobre as plantas de arroz. Nos hospedeiros primários das espécies, as gramíneas nativas de grãos grandes, não há registro da incidência de enxames (BARRIGOSSI, 2008). Os percevejos O. poecilus predominam nos enxames, com poucos O. ypsilongriseus misturados. Essa prevalência de $O$. poecilus já foi reportada por outros autores e também foi constatada no presente estudo (Figura 2). A proporção de $O$. ypsilongriseus chegou, no máximo, a $30 \%$ da população de $O$. poecilus, porém, em média, foi bem mais baixa, em torno de $5 \%$. Couto et al. (2006) verificaram que 0 . ypsilongriseus compreendia apenas $6 \%$ dos percevejos capturados nas armadilhas luminosas em São Miguel do Araguaia (Goiás), ao passo que Fazolin (1993) constatou até $16 \%$ em coletas com rede de varredura em Rio Branco (Acre). Ferreira et al. (2001) argumentam que $O$. ypsilongriseus tem maior afinidade com ambientes secos (arroz de terras altas), ao passo que $O$. poecilus predomina em ambientes úmidos (arroz irrigado).

Interessante observar que no período compreendido entre as safras 2016/17 e 2019/20, em Itajaí, SC, houve uma elevada população de percevejos no final da safra 2017/18, que depois resultou em elevada população inicial na safra 2018/19 (Figura 2). Ou seja, muitos indivíduos (hibernantes) sobreviveram entre essas duas safras. Porém, a alta população inicial da safra 2018/19 não resultou em alta população no seu final e, por consequência, a população na safra 2019/20 foi baixa. Entre outros 

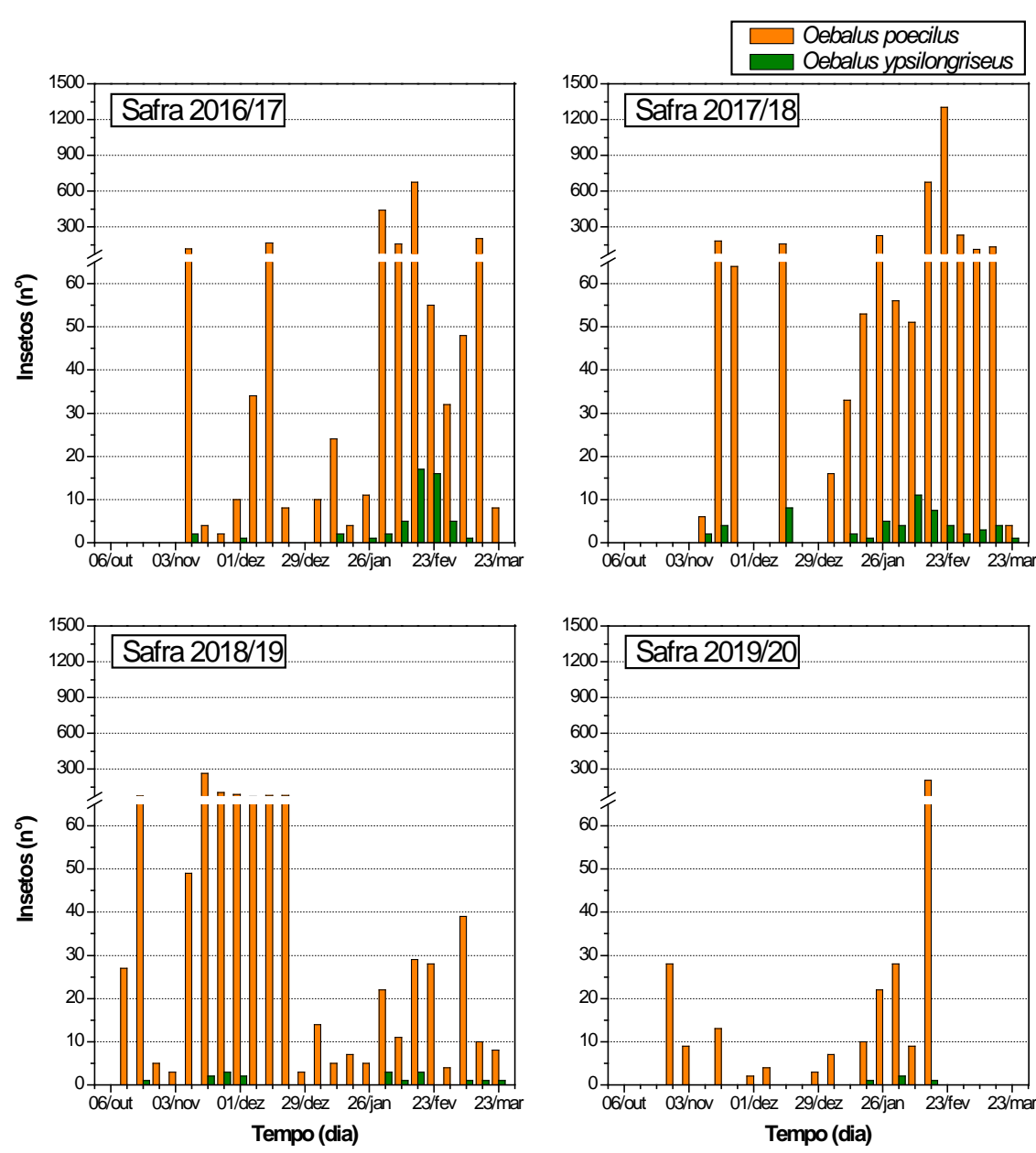

Figura 2. Ocorrência total de $O$. poecilus e $O$. ypsilongriseus em lavouras de arroz irrigado, conforme a safra de cultivo em Itajaí, SC

Figure 2. 0 . poecilus and 0 . ypsilongriseus total occurrence in irrigated rice fields, according to the growing season in Itajaí, SC

fatores, isso pode ter sido devido à ação dos inimigos naturais do percevejo-dogrão, numa clara reação dependente de densidade (BULMER, 1975). Fungos entomopatogênicos e microvespas são os principais agentes de controle biológico dos percevejos Oebalus (FERREIRA et al., 2001; HICKEL et al., 2016). As epizootias fúngicas normalmente ocorrem nas populações de final de safra, eliminando adultos e ninfas antes da formação das populações de hibernantes. Isso resulta depois em baixas populações iniciais na safra seguinte. Microvespas, por sua vez, parasitam os ovos impedindo que uma dada geração deixe muitos descendentes. Assim, por hipótese, apesar da alta população de percevejos hibernados, uma igualmente alta população de microvespas, formada ao longo das safras anteriores, pode ter resultado em altas taxas de parasitismo de ovos, reduzindo o sucesso reprodutivo dos percevejos.

A sucessão entre populações de morfologia hibernal e estival, nas duas espécies de percevejos, ocorre alternadamente (Figura 3). Os percevejos com morfologia hibernal predominam até o mês de dezembro, quando os indivíduos perecem e são sucedidos por aqueles de morfologia estival (a partir de janeiro). A partir de meados de fevereiro, percevejos de morfologia hibernal surgem novamente e passam a compor os enxames, junto com aqueles de morfologia estival. Essas observações corroboram com as de Albuquerque (1993), que verificou no mês de março, no Rio Grande do Sul, a recomposição das po- pulações de hibernantes.

As populações de morfologia estival foram sempre superiores às de morfologia hibernal, refletindo, entre outros aspectos, o suprimento alimentar mais nutritivo no período do verão, que garante maior sucesso reprodutivo às populações de lavoura. Nilakhe (1976) observou que fêmeas de 0 . pugnax, mantidas em plantas de arroz, colocaram duas vezes mais ovos que fêmeas mantidas em outras gramíneas hospedeiras como o capim-arroz (Echinochloa spp.). As populações de morfologia hibernal também estão sujeitas às agruras do inverno, quando muitos indivíduos perecem durante a diapausa (SANTOS et al., 2006).

Hickel et al. (2019) argumentam que a estratégia de controle do percevejodo-grão deveria ser direcionada aos períodos de ocorrência dos indivíduos. Os indivíduos hibernados ocorrem entre novembro e dezembro, meses em que o arroz ainda não está produzindo grãos e, portanto, não está atrativo a esses insetos. Desta forma, os percevejos hibernados buscam outros hospedeiros, 0 que inviabiliza planejar seu controle na lavoura de arroz. Por outro lado, os indivíduos hibernados são aqueles debilitados, que voam pouco e deixam menos descendentes (NILAKHE, 1976) e uma ação de controle sobre essa população teria grande efeito na redução das populações estivais posteriores (HICKEL, 2018). Dessa forma, outras estratégias de manejo integrado deveriam ser implementadas objetivando o controle dos percevejos hibernados, como o cultivo armadilha.

Os enxames de indivíduos estivais ocorrem a partir de fevereiro e estão associados à emissão de panículas pelas plantas. Essa população é a que ocorre no arroz e para qual deveriam ser planejadas as medidas de controle nas lavouras (BARRIGOSSI, 2008, HICKEL, 2018). Contudo, uma maior ou menor população de percevejos, na época dos enxames, depende do sucesso reprodutivo dos indivíduos hibernados (NILAKHE, 1976). Por isso se tornam fundamentais 

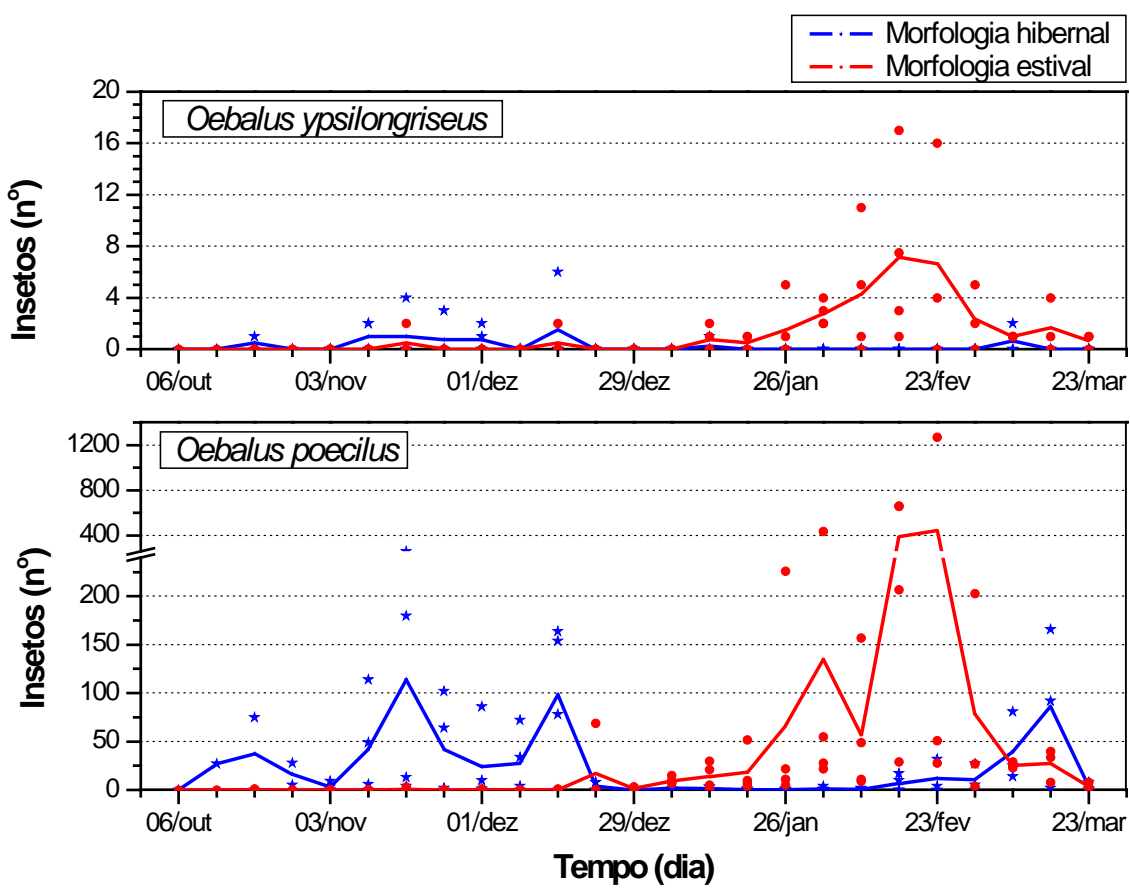

Figura 3. Flutuação populacional média de adultos de $O$. ypsilongriseus e $O$. poecilus de acordo com a morfologia corporal em Itajaí, SC, safras de 2016/17 a 2019/20

Figure 3. Average adult population dynamics of $\boldsymbol{O}$. ypsilongriseus and $\boldsymbol{O}$. poecilus according to body morphology in Itajaí, SC, 2016/17 to 2019/20 seasons

as medidas para conter a população de percevejos hibernados (HICKEL 2018).

\section{Conclusão}

- A flutuação populacional do percevejo-do-grão em arroz irrigado em Santa Catarina se caracteriza por dois momentos de maior incidência de indivíduos, em novembro/dezembro e em fevereiro, correspondendo aos períodos de ocorrência de percevejos hibernados e de percevejos em enxames, respectivamente.

- A espécie 0 . poecilus prevalece sobre a espécie $O$. ypsilongriseus e as populações com morfologias hibernal ou estival ocorrem alternadamente ao longo do período de atividade de voo, se sobrepondo apenas a partir de meados de fevereiro.

\section{Agradecimentos}

À Fundação de Amparo à Pesquisa e Inovação do Estado de Santa Catarina (Fapesc) e ao Conselho Nacional de Desenvolvimento Científico e Tecnológico
(CNPq), pelo suporte financeiro.

Aos acadêmicos de Agronomia Rafael Ducioni Panato, Débora Dal Zotto, Marino Antônio de Quadros, Luciano da Silva Alves e Sérgio Francisco Bervanger pelo auxílio nas coletas e na triagem de insetos.

\section{Referências}

ALBUQUERQUE, G.S. Planting time as a tactic to manage the small rice stink bug, Oebalus poecilus (Hemiptera, Pentatomidae), in Rio Grande do Sul, Brazil. Crop Protection, v.12, n.8, p.627-630, 1993. DOI: https://doi. org/10.1016/0261-2194(93)90128-6

AMARAL, S.F. Biologia e importância econômica do percevejo do arroz, (Solubea poecila Dallas, 1851), no Estado de São Paulo. O Biológico, v.15, n.3, p.47-58, 1949.

ARIAS, E.; GUTIERREZ, Y.A. Oebalus insularis (Heteroptera: Pentatomidae) plaga importante en el cultivo del arroz (Oryza sativa) en Cuba. Havana: CIDA, 1986. 37p. (CIDA. Boletin de Resenhas, 19).

BARRIGOSSI, J.A. Manejo do percevejo da panícula em arroz irrigado. Santo Antônio de Goias: Embrapa Arroz e Feijão, 2008. 8p. (Embrapa. Circular Técnica, 79). Disponível em: https://ainfo.cnptia.embrapa.br/ digital/bitstream/CNPAF-2009-09/28151/1/ circ_79.pdf. Acesso em: 6 abr. 2020.

BULMER, M.G. The statistical analysis of density dependence. Biometrics, v.31, n.4, p.901-911, 1975. DOI: https://doi. org/10.2307/2529815

COUTO, D; BARRIGOSSI, J. A., FERNANDES, P. M. Flutuação populacional de Oebalus spp. (Hemiptera: pentatomidae) no arroz irrigado em várzeas tropicais. In: CONGRESSO BRASILEIRO DA CADEIA PRODUTIVA DE ARROZ, 2., 2006, Brasília, DF. Anais... Santo Antônio de Goiás: Embrapa Arroz e Feijão, 2006. Disponível em: http://ainfo.cnptia.embrapa. br/digital/bitstream/CNPAF/23615/1/ CBCTRAB_71-1.pdf. Acesso em: 10 abr 2019.

DOUGLAS, W.A. Studies of rice stinkbug populations with special reference to local migration. Journal of Economic Entomology, v.32, n.2, p.300-303, 1939. DOI: https://doi. org/10.1093/jee/32.2.300

EBERHARDT, D.S.; SCHIOCCHET, M.A. Recomendações para a produção de arroz irrigado em Santa Catarina (sistema pré-germinado). Florianópolis: Epagri, 2015. 92p. (Epagri. Sistema de Produção, 48).

FAZOLIN, M. Descrição dos danos e dinâmica populacional das pragas que ocorrem na cultura do arroz, em Rio Branco (AC). Rio Branco: Embrapa-CPAF, 1993. 14p. (Embrapa-CPAF. Comunicado Técnico, 59). Disponível em: https://ainfo.cnptia.embrapa.br/ digital/bitstream/item/173392/1/938.pdf. Acesso em: 6 abr. 2020.

FERREIRA, E.; BARRIGOSSI, J.A.F.; VIEIRA, N.R.A. Percevejos das panículas do arroz: fauna heteroptera associada ao arroz. Santo Antônio de Goias: Embrapa Arroz e Feijão, 2001. (Embrapa Arroz e Feijão. Circular Técnica Online, 43). Disponível em: http:// www.cnpaf.embrapa.br/publicacao/circulartecnica/ct_43/ index.htm. Acesso em: 6 abr. 2019.

HICKEL, E.R. Cultivo armadilha para manejo integrado do percevejo-do-grão em arroz irrigado. Agropecuária Catarinense, v.31, n.1, p. 41-44, 2018. Disponível em: file:///C:/ Users/ehick/AppData/Local/Temp/2262301-1-PB.pdf. Acesso em: 06 abr. 2020. 
HICKEL, E.R.; OLIVEIRA, D.G.; EBERHARDT, D.S.; KLEVESTON, R. Flutuação populacional e controle de pragas: estudos de caso com pragas de arroz irrigado. Agropecuária Catarinense, v.32, n.2, p.35-39, 2019. DOI: http:// dx.doi.org/10.22491/RAC.2019.v32n2.2

HICKEL, E.R.; PRANDO, H.F.; EBERHARDT, D.S. Percevejos nas lavouras catarinenses de arroz irrigado: ocorrência, monitoramento e manejo integrado. Florianópolis: Epagri, 2016. 54p. (Epagri. Boletim Técnico, 173).

JONES, D.B.; CHERRY, R.H. Species composition and seasonal abundance of stink bugs (Heteroptera: Pentatomidae) in Southern Florida rice. Journal of Economic Entomology, v.79, n.5, p.1226-1229, 1986. DOI: https://doi.org/10.1093/jee/79.5.1226

KNABBEN, G.C.; CARVALHO, M.W.M.; BERTOLDI, B.; NOVAES, Y.R.; HICKEL, E.R.; HINZ, R.H. Sonne - inovação tecnológica em armadilha luminosa para aplicação no manejo integrado de pragas. Agropecuária Catarinense, v.32, n.1, p.41-44, 2019. DOI: http:// dx.doi.org/10.22491/RAC.2019.v32n1.3
NILAKHE, S. S. Overwintering, survival, fecundity, and mating behavior of the rice stink bug. Annals of the Entomological Society of America, v.69, n.4, p.717-720, 1976. DOI: https://doi.org/10.1093/aesa/69.4.717

NOLDIN. J.A.; HICKEL, E.R.; KNOBLAUCH, R.; EBERHARDT, D.S; SCHIOCCHET, M.A.; SCHEUERMANN, K.K.; KLEVESTON, R.; AGOSTINI, I.; MARTINS, G.N.; MARSCHALEK, R.; WICKERT, E.; ANDRADE, A.; LUCIETTI, D. Recomendações técnicas para a produção de arroz irrigado em sistema orgânico em Santa Catarina. Florianópolis: Epagri, 2015. 40p. (Epagri. Sistemas de Produção, 47).

PANTOJA, A.; GARCÍA, C.A.; DUQUE, M.C. Population dynamics and effects of OebaIus ornatus (Hemiptera: Pentatomidae) on rice yield and quality in southwestern Colombia. Journal of Economic Entomology, v.93, n.2, p.276-279, 2000. DOI: https://doi. org/10.1603/0022-0493-93.2.276

RASHID, T.; JOHNSON, D.T.; BERNHARDT, J.L. Sampling rice stink bug (Hemiptera: Pentatomidae) in and around rice fields. Environmental Entomology, v35, n.1, 102-111,
2006. DOI: https://doi.org/10.1603/0046225X-35.1.102

SANTOS, R.S.S.; REDAELLI, L.R.; DIEFENBACH, L.M.G.; ROMANOWSKI, H.P.; PRANDO, H.F.; ANTOCHEVIS, R.C. Seasonal abundance and mortality of Oebalus poecilus (Dallas) (Hemiptera: Pentatomidae) in a hibernation refuge. Brazilian Journal of Biology, v.62, n.2A, p.447-453, 2006. DOI: https://doi. org/10.1590/S1519-69842006000300010

SUTHERLAND, J.P.; BAHARALLY, V. The influence of weather on the population dynamics of the rice stink bug and the implications for integrated pest management. International Journal of Pest Management, v.49, n.4, p.335-342, 2003. DOI: https://doi.org/10.10 80/09670870310001608653

VIVAS, L.E.C.; NOTZ, A.; ASTUDILLO, D. Fluctuación poblacional del Chinche Vaneadora en parcelas de arroz, Calabozo, Estado Guárico, Venezuela. Agronomía Tropical, v.60,n.1, p.61-73, 2010. Disponível em http://ve.scielo.org/scielo.php?script=sci_ arttext\&pid=S0002-192X2010000300007\&l ng=pt\&nrm=iso. Acesso em: 6 abr. 2020 .

\section{Năo deixe sua consciência escorrer pelo ralo: preserve a água e evite 0 desperdício.}

